\title{
Arbor
}

\section{La imagen de la ciudad y el paisaje urbano ${ }^{1}$}

\section{José Carlos Rueda Laffond}

Arbor CLXIX, 666 (Junio 2001), 303-322 pp.

En contadas ocasiones el tiempo simbólico —el cambio de década, el ecuador de un siglo, el paso de una a otra centuria- adquiere una verdadera relevancia histórica. Más allá del acontecimiento singular, el tiempo histórico se nutre por procesos cuya temporalidad superan una fecha específica. Esto es especialmente evidente si nos aproximamos a los tiempos que convergen en la ciudad. Su escenario es el producto del solapamiento entre transformaciones y pervivencias sometidas a muy distintos ritmos. Además, la definición de su espacio no es sólo la resultante de una suma de alteraciones y cambios materiales lineales, ni su paisaje se reduce sólo a una sucesión de infaestructuras, equipamientos e inmuebles. La ciudad es, ante todo, el producto de unas relaciones sociales, de unas actividades productivas y de unas prácticas políticas.

El Madrid de 1899 no se diferenciaba del Madrid de 1901. Es más, el cambio de siglo parece situarse, si lo interpretamos como punto de inflexión hacia la contemporaneidad, en torno a la segunda década de la centuria. Es alrededor de los años de la Gran Guerra cuando se perfilan con nitidez unas coordenadas demográficas, urbanísticas o productivas más próximas a las que presentan otras capitales europeas ${ }^{2}$. Pero la cronología que pauta el paso de Madrid de Corte a Metrópoli es lenta. Sus límites extremos podrían situarse entre la crisis del Antiguo Régimen y la II República. Desde mediados del XIX se irá advirtiendo el impacto urbano de la desamortización y de la vertebración de un mercado nacional cuyo epicentro se encuentra en la capital del estado liberal. A lo largo de la primera mitad de la década de 1930, el definitivo afianzamiento de la ciudad de las masas. 
La urbe que agrupa nuevos servicios públicos - comerciales, administrativos-, el núcleo donde, por fin, despuntan algunas concentraciones industriales dignas de tal nombre, y que aspira a su futura planificación, es prácticamente coétaneo al Madrid circunscrito entre el 14 de abril y el 18 de julio ${ }^{3}$.

¿Qué papel ocupa 1900 en este proceso? En 1929 el Ayuntamiento de Madrid publicaba las Bases para un Concurso Internacional dirigido a planificar el futuro Madrid ${ }^{4}$. El Premio del Concurso quedó desierto. No obstante, de aquel esfuerzo se derivaron dos textos básicos para entender los perfiles de la ciudad. Por un lado, el anteproyecto presentado por los arquitectos Secundino Zuazo y Hermann Jansen, un estudio que pretendía una completa reforma de la urbe, una profunda revisión de su sentido como «comunidad económica, social y política» ${ }^{5}$. Esta reflexión supuso la culminación en un esfuerzo por redefinir Madrid y sus pautas de crecimiento. Un esfuerzo teórico y técnico que se había desarrollado a lo largo de los tres decenios anteriores, donde participaron nombres como Soria, López Sallaberry, Núñez Granés, Salvador, Aranda, Lorite o Anasagasti. Por otro lado, gracias a la convocatoria del Concurso la Oficina Municipal editó una ambiciosa Información sobre la ciudad ${ }^{6}$. Este voluminoso trabajo resultó mucho más que una foto fija del Madrid de finales de los años veinte. A partir de una recopilación exhaustiva de diversos materiales, recogió pormenorizadamente los rasgos presentes en la evolución demográfica, productiva, urbanística o cultural a lo largo de todo el primer tercio del siglo XX. La Información fue, por tanto, un compendio de los problemas urbanos heredados y, asimismo, de las realizaciones espaciales y de sus contradicciones a lo largo de dicha etapa.

Podríamos estimar que aquel texto reflejaba los trazos históricos de una ciudad que se había caracterizado por la transición, inconclusa y desajustada, hacia la modernidad. El Madrid de 1900 debe interpretarse a la luz de ese proceso. La capital era, en el cambio de siglo, muchas cosas: la residencia de la Corona, de sus elites de poder y de su maquinaria administrativa; la población donde convivían los oficios tradicionales y una fragmentada trama mercantil; el puerto de llegada para una emigración creciente, una verdadera legión de futuras criadas u obreros de la construcción... Pero la ciudad estaba perfilando también —en sus actividades y espacios, en sus ámbitos culturales y de esparcimiento, en las actitudes y los comportamientos públicos y privados- el alumbramiento de la sociedad de masas. Alumbramiento que no será sinónimo de pleno aquilatamiento de clases compactas. $\mathrm{Y}$ que tampoco resultará un obstáculo para la pervivencia de una 
estructura social heterogénea, donde confluyen grupos, actividades y mentalidades diversos, y, con ellos, dialécticas muy distintas que incidirán, y se verán condicionadas, por el escenario urbano.

Este trabajo se aproxima a dos dimensiones desde donde podemos situar las claves que conforman la imagen y el paisaje de la ciudad en 1900. Por un lado, repasaremos esos rasgos - demográficos y habitacionales, productivos, simbólicos, políticos...- que condicionan las características de la trama urbana y sus contradicciones. En segundo lugar, nos acercaremos a algunos aspectos que nutren la política urbanística en torno al cambio de siglo y oponen la reforma del casco interior con la planificación de su futura expansión. Ambas líneas se encarnan, respectivamente, en los proyectos de la Gran Vía y del ordenamiento del Extrarradio.

\section{Madrid, 1900: las dialécticas del paisaje urbano}

Madrid, en torno a 1900, ofrecía, al menos, cinco retos urbanos que se verán agudizados en los decenios siguientes: el generado por las pautas dictadas por el incremento demográfico, el que se deriva de su heterogéneo universo social y de su reparto en el espacio urbano, el relativo al impacto de las actividades productivas sobre el paisaje, el derivado de la inexistencia de referentes simbólicos y, finalmente, la contradicción establecida entre los objetivos regeneracionistas y los límites impuestos por la política municipal.

No es éste el lugar para señalar pormenorizadamente las claves demográficas del Madrid de 1900. Recordemos, tan sólo, que en los primeros años de siglo pervive un crecimiento vegetativo negativo, sólo corregido por la recepción de un abultado contingente de emigrantes. Este segundo factor apunta un rasgo que no hará sino amplificarse durante todo el primer tercio del siglo: el del espectacular incremento de su población, que duplicará su volumen, y alcanzará el millón de almas en 1930. Además, en 1900, se mantienen las características tradicionales de dicho éxodo. Está nutrido básicamente por población rural, por cohortes dirigidas al servicio doméstico o a la construcción, que proceden de las provincias limítrofes - Guadalajara, Toledo, Segovia...-, y que con frecuencia se afincan en la capital de forma estacional. Su repercusión sobre el caserío es clara, dado que aporta, en buena medida, el componente humano que habrá de concentrarse en las barriadas incontroladas de la periferia ${ }^{7}$. 
Aunque se ha señalado un equilibrio en la ratio habitantes por vivienda entre 1900 y 1935 —en torno a un 4'6 y 4'2 para las dos fechas citadas-, y un incremento paralelo entre la relación de habitantes y viviendas por inmueble ${ }^{8}$, es indudable que desde 1900 también se agudizaron las diferencias por barrios. Así, por ejemplo, dos distritos del Interior como Centro y Latina manifestaron tendencias contrarias. El primer caso es el del único distrito que ofrece un crecimiento poblacional negativo, disminuyendo el número de sus vecinos en 7.464 personas entre 1910 y 1930. Tomando como referencia los años 1905 y 1920 el monto de sus viviendas se reduce también en casi un millar, pasando de 13.089 a 12.453. El distrito de Latina, que incluye alguna de las barriadas más deprimidas del caserío madrileño, sufre, por el contrario, un incremento de más de 20.000 vecinos entre 1905 y 1920, mientras que el número de viviendas (15.105 y 15.865 respectivamente) se mantiene inalterado ${ }^{9}$.

En torno a las barriadas extremas de Latina encontraremos en 1900 al lumpen madrileño. No obstante, el universo social en la ciudad está muy lejos de su articulación mediante clases puras, y su complejidad dota de sentido a una segunda dialéctica urbana. Es más, si bien podemos hablar de un Madrid burgués, definido en torno al Ensanche -en los distritos de Buenavista o Congreso, en los barrios de Almagro y Salamanca-, esta dimensión no es sino un componente peculiar respecto a una estructura social donde aún se mantienen, dominantes, los trazos protoindustriales presentes treinta o cuarenta años antes: una base popular sobredimensionada, un sólido apartado de funcionarios y militares, un volumen raquítico de profesionales, los omnipresentes estratos de pequeños y medianos comerciantes o fabricantes... Una trama social muy alejada, en definitiva, de un hipotético escenario que pueda ser sometido a rígidas pautas de zonificación.

A estos epígrafes hay que sumar, desde luego, el dispar capítulo de propietarios, categoría que engloba desde el noble de viejo cuño hasta el burgués rentista. Y debe recordarse la figura del casero madrileño, que en su praxis histórica encarnará una verdadera antítesis del promotor inmobiliario. No debemos olvidar, en este sentido, frente a las ideas recogidas en el Anteproyecto para el Ensanche de Carlos María de Castro (1860) -la expansión urbana planificada, la introducción de criterios de segregación residencial, productiva y «buen gusto»-, el fracaso empresarial del Marqués de Salamanca, tras la profunda crisis abierta en el negocio de la construcción en $1866^{10}$.

El Ensanche creció lenta y desordenadamente a lo largo del último tercio del siglo XIX. Según los datos elaborados por la Comisión de Ensanche del Ayuntamiento, entre 1868 y 1878 se edificaron 351 nuevos 


\section{La imagen de la ciudad y el paisaje urbano}

inmuebles en esta zona, entre 1879 y 1889,738 , entre 1889 y 1899 , 424, y, entre 1900 y 1910, 715. Por otro lado, el Plan Castro se vio tan profundamente alterado que requirió ser revisado por un nuevo plano formulado en 1900. En este texto, que por fin adquirió la categoría de proyecto, los arquitectos municipales Francisco Andrés Octavio y Eugenio Jiménez Correa incluyeron distintas alteraciones introducidas desde los años sesenta y revisaron su trazado. Además, todavía en este año de 1900, el caserío madrileño de nueva planta dependía, casi abrumadoramente, de iniciativas personales atomizadas - muchas veces pendientes de prácticas especulativas a corto plazo-, y no de proyectos de promoción inmobiliaria de carácter sistemático. Como excepción únicamente podrían recordarse, en torno a estos años del cambio de siglo, empresas caritativas de desigual fortuna, como las promovidas por La Constructora Benéfica, la Sociedad Benéfica Española, el Montepío General o La Vivienda Mutualista.

Y, por supuesto, el empeño aislado de Arturo Soria y su Ciudad Lineal. En 1894 Soria constituyó la Compañía Madrileña de Urbanización como marco empresarial para promover su proyecto urbanístico de una ciudad lineal. Contemporánea a las enseñanzas de Sitte, y cercana a las ideas de ciudad-jardín de Howard, Soria establecía unos planteamientos de crecimiento y ordenamiento radicalmente contrarios a las pautas dictadas por el urbanismo finisecular más ortodoxo. Su ciudad se configuraría a partir de un eje viario ilimitado. Esta idea llevaba consigo no sólo el esfuerzo de promoción inmobiliaria más importante en el Madrid intersecular, sino que además superaba los límites técnicos, ideológicos y, por supuesto, urbanísticos, sobre los que se apoyaban las directrices municipales relativas a la cuestión de la futura expansión de la capital española ${ }^{11}$.

Por otro lado, Soria se hacía eco de unos criterios que se acabarán convertiendo en verdaderos parámetros de su proyecto. La racionalidad como emblema de la modernización urbana, las necesidades de salubridad e higiene, la lógica dictada por la funcionalidad, el lograr un óptimo equipamiento en infraestructuras y servicios... Todo ello son los ejes para una empresa alejada de rígidas pautas zonificadoras. «La posibilidad de que todos, ricos y pobres, vivan en terreno y casa de su propiedad, suntuosa en unos casos, modestísima en otros» es el horizonte del proyecto. Un horizonte que, desde luego, está conformado por los mitos cooperativistas finiseculares, por los ideales democráticos dirigidos a que «la clase obrera (obtenga) los medios de habitar y adquirir la propiedad de una casa mediante el concurso del trabajo individual, del ahorro y de la previsión ${ }^{12}$. 
La Compañía Madrileña de Urbanización promovió en estos años un abanico diversificado de servicios. La sociedad inmobiliaria se implicó en organizar el transporte y el suministro eléctrico a la barriada, y disponía de una imprenta donde se publicó La Ciudad Lineal, el periódico de la compañía, infatigable proselitista de sus bondades sociales y de la innovación urbanística. No obstante, esta diversificación tocará fondo en 1914, momento en que la creciente acumulación de gastos fijos apenas se equilibra ya por unos ingresos dubitativos. Bastará una contracción en estos últimos, provocada por algunas retiradas de capital en la Caja de Ahorros de la C.M.U., para que todo el entramado se resienta y se fuerce la suspensión de pagos.

Una tercera línea de tensión que incide sobre los perfiles del paisaje urbano se descubre a partir del difícil acoplamiento que tienen en la ciudad las diferentes actividades productivas. Paradigma de ciudad imperial, tal y como la definiera en su día Jan de Vries, Madrid mantiene en $1900 \mathrm{su}$ impronta como núcleo administrativo. No obstante, es en los decenios interseculares cuando podemos apreciar la definición de áreas industriales que apenas, eso sí, salpican desordenadamente algunos puntos del Ensanche, en torno a Cuatro Caminos o, sobre todo, en los aledaños de las estaciones de Atocha, Delicias e Imperial ${ }^{13}$. Tampoco hay, tampoco puede haber, un diseño urbanístico que planifique decididamente la diferenciación espacial según criterios de especialización económica. Otro tanto ocurrirá, incluso, con la traumática oposición que enfrentará, a partir de 1910, los nuevos servicios localizados en la Gran Vía y el universo secular de espacios y actividades nucleados en torno a la Puerta del Sol. En efecto, la nueva arteria actuará como pantalla, tras la cuál pervive un tejido urbano tradicional, muy anterior a los nuevos usos terciarios que se irán emplazando en la arteria.

Evidentemente Madrid no era ni Londres ni Manchester. Pero, no obstante, si bien hemos de hablar de un paisaje económico que asiste a lentas transformaciones, han de señalarse también los efectos inducidos por su caracterización como capital económica del estado liberal a partir del segundo tercio del siglo XIX. Capitalidad económica que, desde luego, se traduce en su impronta como punto de convergencia, intercambio y consumo dependiente de una red de transportes y comunicaciones paulatinamente más integrada. Red abierta hacia el exterior, que se completará con el diseño, a lo largo del cambio de siglo, de sus transportes interiores ${ }^{14}$. En efecto, en 1899 se constituyeron en París las dos sociedades que explotaban las empresas madrileñas de tranvías (Este, Estaciones y Mercados, General, Madrileña y Norte). 
Y si bien habrá que esperar hasta octubre de 1919 para asisitir a la apertura de la primera línea del metropolitano, las ideas sobre un ferrocarril subterráneo se multiplicaron desde la década de los noventa. Incluso los primeros bosquejos elaborados por Mendoza, González Echarte y Otamendi se habían fechado ya en torno a 1904.

Una cuarta dimensión desde donde hemos de interpretar el paisaje urbano del Madrid de 1900 es la referida a la articulación de espacios simbólicos. La revolución liberal trae consigo el Ateneo y la Universidad. Madrid es capital cultural y periodística indiscutida a lo largo de la segunda mitad del siglo XIX ${ }^{15}$. Pero es incapaz de articular espacios representativos capaces de resumir formalmente, y sacralizar, su nueva impronta capitalina. Desde una perspectiva peculiar - la del liberalismo democrático-, Angel Fernández de los Ríos había valorado sobremanera este componente en su Futuro Madrid. Ya en la Restauración, y muy particularmente alrededor del cambio de siglo, casi todos los empeños por dotar a Madrid de los rasgos simbólicos que se considera han de escenificar al nuevo régimen se verán abocados al fracaso. En este sentido, no es exagerado apuntar que la capital española acaba constituyendo un verdadero contrapunto al París del II Imperio o a la Viena fin de siglo. Recuérdese, por ejemplo, la extrema lentitud, y definitivo abandono, de las obras de la Catedral de la Almudena. Y el olvido de las diversas intervenciones previstas sobre el centro de la ciudad, en los aledaños de Palacio, como el proyecto presentado en 1903 por Miguel Mathet con el propósito de realizar un bulevar entre las calles de Mayor y Arenal ${ }^{16}$. Otro tanto ocurrirá con la construcción del nuevo templo de Atocha. A pesar de tratarse de una idea impulsada en 1890 por la Regente, la nueva basílica -que debería haberse convertido en el recinto sacro y político por excelencia de la capital- no se levantará hasta años después de la guerra civil. De todos los proyectos barajados en torno a 1900 únicamente se realizarán dos obras: el Panteón de Hombres Ilustres, que debió ser claustro neobizantino de la nueva basílica de Atocha, obra de Fernando Arbós (1897-1899); y el grupo monumental en honor a Alfonso XII, presentado en 1901 y finalmente erigido en el Retiro.

La definición de estos espacios simbólicos con innegable carga política convergieron, en los años interseculares, con otros propósitos mucho más vastos. En efecto, la necesidad de transformar Madrid fue una idea omnipresente para higienistas, políticos o periodistas. Además, siempre se presentó mediante un lenguaje y unas propuestas derivadas de lo que podríamos interpretar como una lógica regeneracionista. No era extraño, por tanto, que la reforma urbana se inscribiese en un 
contexto de mejora integral, donde debían ligarse otras transformaciones complementarias, tanto en el plano espacial como en el habitacional o el higiénico ${ }^{17}$. Tales propuestas ocasionalmente superaron los límites marcados por la Ley Municipal de 1877, texto que hacía de los Ayuntamientos el último peldaño en la organización territorial de un Estado centralista articulado en clave moderada ${ }^{18}$. El fomento de las mejoras viarias, la urbanización de los suburbios, la política de casas baratas o respecto al abastecimiento de artículos de primera necesidad, se constituyeron en objeto de estudio $\mathrm{y}$, puntualmente, en materia de iniciativa legal. Pueden recordarse, en este sentido, desde la Ley de mejora y saneamiento de 1895 hasta las primeras disposiciones sobre casas baratas dictadas en 1911 . Y es en este marco donde se debe localizar también el debate sobre la municipalización de servicios urbanos y la revisión de las competencias económico-administrativas cedidas al Consistorio. Abogando por las ideas retóricas de la regeneración urbana, confluyeron argumentos de muy distinto tinte (liberalizadores, intervencionistas, cooperativistas:..), en lo relativo a cuestiones como el «industrialismo municipal» o el fomento de iniciativas municipalizadoras ${ }^{19}$.

Por otro lado, a la altura de 1900 el repertorio de problemas urbanos era, en buena medida, similar en las principales capitales europeas. Su relación era amplia: problemas de degradación del hábitat, un crecimiento humano desmedido, encarecimiento y carestía de las viviendas o la sucesión de crisis y conflictos donde se combinaba desde el motín a la huelga. Semejantes tensiones indujeron, incluso de manera espontánea, a la alteración de sus fisonomías. También a la reafirmación acerca de la necesidad de una intervención guiada desde los poderes públicos que transformase los núcleos históricos y dictase sus futuras expansiones. El cambio de siglo supo articular un referente común para los elementos que incidieron en los distintos debates urbanos. Éste fue el de la modernidad. No es casual que Antonio Pedrini presentase en 1905 un trabajo titulado La cittá moderna como compendio de las bondades sociales de la «ingenieria sanitaria» y de la técnica, claves que consideraba como auténticas impulsoras de las mejoras de la urbe. Tampoco lo es que Otto Wagner reivindicará en su Arquitectura Moderna (1895) racionalidad y funcionalidad como premisas para ordenar un ámbito que surgía como consecuencia de los cambios sociales.

Mucho antes del decenio de los noventa se había reiterado en la urbanística europea la necesidad de un Plan General como exposición coherente de las pautas de intervención. Entre 1858 y 1862 se estableció un primer estadio de la información y el debate urbano a la sombra 
del Plan Regulador de Berlín de Hobrecht. Y antes de que arrancase la nueva centuria, se habían concitado diversas propuestas - recuérdese los nombres de Huber, Faucher, Broch, Dohna-Poninski, Fritsch, Orth, Eberstadt o Wolf-, y se habían multiplicado los planos generales en varias ciudades alemanas. Empero, la mejor síntesis de lo producido en venticinco años de reflexión teórica y de práctica urbana vio la luz en 1890, gracias a la pluma de Joseph Stübben. Der Städteban se convirtió, hasta la Primera Guerra Mundial, en el tratado por excelencia. Supo resumir las pautas que guiaban la reforma y racionalización de la ciudad. Y constituyó el mejor reflejo de ese cientifismo social que asumía a la ciudad como globalidad y como resultante de problemas en buena medida autónomos, pero interrelacionados.

Figura ambivalente del escenario político —cabeza visible en la renovación del liderazgo del Partido Liberal; encarnación, del arribismo gracias a las maniobras y a las fidelísimas clientelas políticas-, Alvaro de Figueroa ocupó la Presidencia del Ayuntamiento en 1898. Y encarnó, contradictoriamente, la figura del munícipe reformista ${ }^{20}$. Un buen ejemplo de este perfil lo encontramos en la defensa retórica del proyecto de la Gran Vía. Para Romanones, la gran avenida que alteraría la fisionomía del Interior se justificaba por «las demandas reiteradas de la opinión pública». Con esta obra no se trataba sólo de mejorar las comunicaciones y sanear parte importante del entramado viario de la ciudad. El Ayuntamiento facilitaría con ella una "provisión de obras» a largo plazo que paliaría «la gran crisis económica» que afectaba «a las clases obreras en su expresión más rudimentaria, a todas las artes, todos los oficios y todas las industrias» ${ }^{21}$.

Otro ejemplo emblemático de munícipe reformista lo encontramos en Alberto Aguilera, Alcalde de Madrid entre 1901-02 y 1906-07. Miembro del Partido Liberal, formó parte de la clientela política de Moret y ocupó un buen número de cargos durante el Sexenio y la Restauración: diputado en Cortes, varias veces Gobernador Civil - de Madrid entre 1898-99 - y Ministro de Gobernación en el Gabinete Sagasta de 1894. Aguilera defendió la necesidad de un ambicioso proyecto de reformas durante su paso por el Consistorio de la capital. Un programa que no sólo incluiría una relación de obras públicas, sino la argumentación de que, desde los poderes públicos, podía incidirse en la mejora social: en el «embellecimiento de la población», en "el mejoramiento y la baratura de los artículos para la vida» y en el «aumento de trabajo para las clases obreras» ${ }^{22}$.

Todas estas propuestas chocaron con los límites, políticos y presupuestarios, del Ayuntamiento madrileño. Esta última dialéctica también 
condicionó, por supuesto, los rasgos del paisaje urbano de 1900. La dependencia respecto al Gobierno de cada Alcalde de la Villa y Corte, la mala administración, los abusos ante los vecinos, la falta de un programa de intervenciones realista, las poderosas tramas conformadas por los intereses y dependencias surgidas del caciquismo urbano... Todo ello son elementos que limitan la eficacia municipal ${ }^{23}$. No es extraño, por ejemplo, que en la resolución publicada en 1896 por el Consejo de Estado a raíz de las denuncias presentadas por varios propietarios del Ensanche contra el Consistorio, dictaminase la anulación de expedientes, apuntando incluso que «es tal el número de infracciones legales cometidas, que puede afirmarse son tantas como actos ejecutados por la Comisión y Corporación Municipal». A todos estos problemas habrá que añadir, por supuesto, las trabas presupuestarias. Las cuentas ordinarias de 1901 apenas si superaron los treinta millones de pesetas. Cantidad insuficiente no sólo ya para emprender ningún programa extraordinario de reforma, sino siquiera para ejecutar una relación sumaria de obras. Las partidas dedicadas a obras públicas presentaban un precario porcentaje «en torno a un diez por ciento del total de gastos previstos», con lo cuál únicamente podrían cubrirse trabajos de mantenimiento. Por el contrario, una importantísima proporción de los ingresos presupuestados habría de dirigirse a cubrir los intereses y la amortización de cargas, como la deuda municipal y el contingente provincial.

Un ejemplo emblemático de la desidia e incapacidad municipal lo encontramos en el fracaso para ejecutar los trabajos de canalización y saneamiento del río Manzanares. Los primeros pasos encaminados a convocar un concurso de proyectos con tal fin tienen lugar en 1900 , durante el mandato del Duque de Santo Mauro. Tan sólo se presentará un trabajo, el del arquitecto Mauricio Jalvo. En él se recogían ya las ideas referidas al encauzamiento, la urbanización de un paseo ribereño y la construcción de presas de compuertas. El texto fue aprobado en julio del año siguiente. Las dificultades para contratar las obras fuerzan un nuevo proyecto, y en enero de 1904 el Consistorio acuerda provisionalmente la concesión de obras al propio Jalvo. Apenas doce meses después Arraga, Mariano Belmas y el ingeniero británico C.S. Meilk dirigen al Ayuntamiento un proyecto alternativo sin presupuesto. Y en enero de 1906 se opta por la convocatoria de un segundo concurso. Jalvo, con el apoyo del Marqués de Santillana, propondrá un nuevo texto. No obstante, la Corporación nunca emitió dictamen. En julio de 1908 un grupo de diputados madrileños presentarán al Congreso una Proposición de Ley para solicitar al Gobierno que costease las obras. 
Se convocará un tercer concurso, que quedará desierto. Estamos en pleno conflicto eléctrico en la capital, en el momento en que se enfrentan los intereses de Hidráulica Santillana y el Canal de Isabel II. Finalmente, en Consejo de Ministros celebrado en febrero de 1910 se resolvió encargar a la recién creada Jefatura de Canalización del Manzanares un proyecto definitivo. A mediados de 1914 se subastaron los trabajos de canalización, y a la altura de finales de los años veinte únicamente se habían instalado dos colectores generales y se había procedido a algunas obras de encauzamiento.

\section{Reforma versus expansión: la Gran Vía y el proyecto de urbanización del Extrarradio.}

En 1882 el Ayuntamiento madrileño confeccionaba un primer borrador de intenciones acerca de la apertura de una o varias grandes vías en Madrid, orientadas fundamentalmente a mejorar la comunicación interior en la ciudad y sus condiciones higiénicas. El arquitecto municipal Pedro Domínguez presentó un proyecto muy vago sobre dos posibles vías interiores en dirección norte-sur, con un coste aproximado de venticinco millones de pesetas. Veinte años antes, el Municipio madrileño ya había aprobado la realización de obras para el ensanche en la calle de Preciados. Por su parte, en 1886 el arquitecto Carlos Velasco publicó las líneas básicas de un anteproyecto para una Gran Vía Transversal que, con tres alineaciones, uniría la Corredera Baja de San Pablo con la calle del Alamo y la de Leganitos. Para ello preveía la expropiación de un total de 312 inmuebles ${ }^{25}$.

Podemos considerar estos tres proyectos como los antecedentes directos de la Gran Vía madrileña, la obra más emblemática realizada en Madrid a comienzos de siglo. La apertura de grandes arterias en el centro de la ciudad constituye uno de los rasgos característicos en la urbanística europea de la segunda mitad del siglo XIX, trasladando la idea del grand boulevard como eje descongestionador sobre el viejo emplazamiento urbano. Representa, por tanto, una formulación complementaria a los criterios decimonónicos de expansión planificada de la urbe, aún encarnados, en el caso madrileño, en la parsimoniosa y discontinua ocupación del Ensanche. Incluso se argumentará que la gran vía puede interpretarse, como principio, en forma de ensanche viario, materializándose por ejemplo mediante la ampliación de ejes longitudinales ya existentes, como los paseos de Delicias, Prado, Recoletos y Castellana ${ }^{26}$. No obstante, la dilatadísima tramitación del 
expediente de la Gran Vía -entre 1898 y 1910-, y el inicio definitivo de los derribos a partir de la última fecha suponen también una operación económica que se llevará a cabo en detrimento de otras opciones manejadas por la política municipal. El espaldarazo final al proyecto contrasta vivamente así no sólo con la incapacidad consistorial para emprender, en paralelo, la ingente tarea de sanear y proyectar la urbanización del Extrarradio. También con la posibilidad de formular un Plan General de Reforma que pueda ser financiado a corto plazo, y donde se recoga una amplísima relación de operaciones urbanísticas $\mathrm{y}$ de mejora funcional.

En marzo de 1895 se había promulgado la Ley sobre obras de saneamiento y mejora interior para las poblaciones con más de 30.000 habitantes. En este texto mejoraban las condiciones de concesión y se amplaban los terrenos susceptibles de ser expropiados. Al mismo tiempo, se contemplaba la posibilidad de que los Consistorios recurriesen a fondos extraodinarios para la financiación de los trabajos. Este marco legal, y el interés municipal entre 1897 y 1906, permitieron la definitiva formulación del proyecto de la Gran Vía. Dos técnicos municipales, José López Sallaberry y Francisco Andrés Octavio, fueron encargados de revisar y ampliar el trazado previsto en 1862 para el ensanche de Preciados. Pero, en realidad, su idea recuperaba buena parte de la concepción de Carlos Velasco, y concluía en una ambiciosa operación por la cual se reformaba la prolongación de la calle Preciados, se enlazaba con Callao y ésta, con Alcalá ${ }^{27}$. Después de una dilatadísima tramitación, por medio de las Reales Ordenes dictadas en agosto de 1904 y enero de 1905, se procedió a la definitiva sanción gubernamental del trazado. Éste se resolvía mediante una gran avenida, dividida en tres secciones (Alcalá-Red de San Luis-Callao-San Marcial), con un ancho de entre 25 y 35 metros en su tramo central y un largo definitivo de poco más de un kilómetro. El alcance de la remodelación repercutiría profundamente sobre el trama viaria del Interior de la urbe: iban a expropiarse y demolerse 358 fincas, desaparecerían 19 calles, otras 32 alterarían parte de su trazado y, entre 1910 y mediados de los años cincuenta, se edificarían 83 nuevos inmuebles.

Hasta el ecuador de la primera década del nuevo siglo el proyecto de la Gran Vía formaba parte, asimismo, de un abanico de operaciones de saneamiento y mejora del Interior que, como idea general, nunca fue llevado a cabo. Cómo se ha señalado en páginas precedentes, Alberto Aguilera fue su mejor valedor. Además de algunas obras puntuales previstas en el distrito Centro, como el proyecto de Miguel Mathet de 1903, el Municipio consideró que el futuro programa de reformas 


\section{La imagen de la ciudad y el paisaje urbano}

habría de incluir también otras intervenciones, como las relativas a la construcción de la Necrópolis del Este, el saneamiento del alcantarillado, el colector para el Manzanares, la ampliación y adecentamiento de mercados y mataderos, o la mejora de las casas de socorro, centros escolares y otras dependencias municipales. La presentación, en 1905, de un Anteproyecto de Plan General de Reforma de Madrid parecía llamado a cubrir tales expectativas y culminar incluso proyectos previstos desde finales del XIX, a pesar de que todavía no se trataba de un plan sistemático de actuación. El texto, sin embargo, nunca fue aprobado por el Consistorio.

Durante la segunda presidencia de Aguilera, en 1906, se insistió de nuevo en fijar un Plan General dedicado a «atender las líneas del Madrid del porvenir». Además, se preveía una provisión extraordinaria de fondos susceptible de impulsar el inicio de las obras. El coste global de la operación sumaba alrededor de cincuenta millones de pesetas, cuantía imposible de cubrir desde las cuentas municipales ordinarias ${ }^{28}$. Dos de los trabajos técnicamente mejor perfilados, y que habían encontrado ya una mayor resonancia entre la opinión pública - la Gran Vía y la Necrópolis del Este-, no se incluyeron en este fallido plan de financiación. Ambos habrían de resolverse mediante sendos proyectos extraordinarios en 1907 y 1908.

El elemento esencial que provocó la dilación en el comienzo de las obras había sido el relativo a las dudas financieras que arrastraba el proyecto. Esta cuestión resultó un aspecto omnipresente en el debate que, durante los primeros años del nuevo siglo, se mantuvo en los medios madrileños acerca de la conveniencia de iniciar los trabajos de la nueva arteria. El cálculo y pago del coste de las expropiaciones - estimadas en cerca de 42 millones de pesetas-, y el abono por parte del Consistorio de la diferencia prevista en el presupuesto entre los ingresos y los gastos, valorada en la misma fecha en cerca de nueve millones de pesetas, entorpecieron la concesión de los trabajos. Tras tres subastas infructuosas, y agotados los plazos legales para su contratación, el Ayuntamiento madrileño se embarcó en 1908 en una ambiciosa operación de endeudamiento con la que se asegurarían poco más de quince millones de pesetas ${ }^{29}$. Trasgrediendo la mecánica de concesión prevista por la Ley de 1895, en diciembre de aquel año se verificó una cuarta subasta, adjudicándose provisionalmente la contrata de las obras al industrial y periodista guipuzcoano Rafael Picavea.

El abandono de Picavea antes de la concesión definitiva desbarató la posibilidad de comprometer capital español en el proyecto y forzó una revisión técnica y financiera del pliego de condiciones ${ }^{30}$. Sin em- 
bargo, antes de la renuncia de Picavea el Ayuntamiento madrileño había recibido una proposición del banquero francés Martin Albert Silber sobre la base de alterar la forma de adjudicación. El Municipio se haría cargo del coste total de las expropiaciones y se convocaría, no una quinta subasta, sino un concurso público ${ }^{31}$. Aceptadas las condiciones, en el verano de 1909 Silber ingresaba una fianza provisional en el Credit Lyonnais, garante del acuerdo. La cantidad tipo del concurso se fijó en treinta millones de pesetas, manteniendo el cálculo previsto respecto a las expropiaciones. Éstas se abonarían mediante los ingresos extraordinarios previstos en el empréstito municipal de 1908 y gracias a un anticipo cedido por el concesionario. Finalmente, en abril de 1910 se iniciaron los derribos, tras una solemne ceremonia que contó con la presencia de Alfonso XIII. Nicolás de Peñalver -alcalde de la capital entre octubre de 1907 y octubre de 1909, impulsor del empréstito extraordinario y representante del adjudicatario desde noviembre de aquel año-, dará su nombre al primer tramo de la nueva arteria.

El mismo año del inicio de las obras de la Gran Vía, el técnico municipal Pedro Nuñez Granés hizo público su proyecto para la urbanización del Extrarradio, el espacio circunscrito entre el casco urbano y los límites del Municipio. Este trabajo formará parte de un dilatado debate, que en puridad se trasladará hasta los años treinta, referido a los contenidos e instituciones que debían comprometerse en el saneamiento y urbanización de este área, donde se encontraban los emplazamientos marginales más importantes de la capital. A la altura de 1915 se estimaba en 50.000 personas el volumen de residentes en el Extrarradio, que carecía de planificación viaria y disponía de unos servicios urbanos muy precarios. Pero, paradojicamente, el Ayuntamiento concedía licencias de construcción en la zona. En torno a 1900 podemos considerar que un tercio de las edificaciones madrileñas estaban emplazadas en esta área. No obstante, el proyecto de Nuñez Granés iba a tener un final muy distinto al de López Sallaberry y Andrés Octavio. No fue aprobado por el Ministerio de Gobernación hasta 1918, y quedó desestimado por el propio Municipio al elaborarse, ya en 1923, un nuevo Informe sobre la urbanización del Extrarradio por parte de los arquitectos López Sallaberry, Aranda, Lorite y García Cascales.

Sin embargo, las primeras iniciativas intervencionistas sobre el Extrarradio habían sido muy anteriores. Ya por Real Orden de agosto de 1888 se había establecido la necesidad de que el Ayuntamiento estudiase el trazado de las futuras vías en la zona. Y en 1896 se propuso que «con sujección a la vigente Ley de Ensanche interior de 
las grandes poblaciones, (se dispusiera) que la junta de Urbanización estudie un plan de reformas del interior de Madrid, teniendo presente las aprobadas por el Ayuntamiento, y otro de urbanización de su término municipal sobre la base del plano del Ensanche en un radio que no exceda de ocho quilómetros, a partir de la Puerta del Sol» ${ }^{32}$. Después de sucesivos informes infructuosos, el Ayuntamiento constituyó en junio de 1906 una Comisión Especial encargada de los preliminares para lo que debía ser «un plan general para la urbanización del Extrarradio y un proyecto de Ley para el mismo que le diera una reglamentación análoga a la del Ensanche». Por fin, en 1908, acordó la creación de una Sección especial encabezada por el ingeniero Pedro Nuñez Granés, director de Vías Públicas. Y entre ese año y finales de 1909 fue redactado el proyecto, convirtiéndose desde entonces, y hasta los primeros años veinte, en el referente inexcusable de una polémica que va a enfrentar concepciones divergentes acerca del propio concepto de la ciudad ${ }^{33}$.

El proyecto de Nuñez Granés preveía la urbanización de una superficie de aproximadamente 466 hectáreas, circunscrita entre los límites del Ensanche - que se veía rectificado en algunos puntos- y una «Gran Vía-Parque» de un largo de 27 kilómetros y de cien metros de anchura. La primera estimación de las expropiaciones a cubrir por el Municipio alcanzaba los tres millones y medio de pesetas. El trazado de la misma se resolvía a partir de una estricta acotación del terreno en cuatro grandes zonas de actuación, en las que debía tenerse en cuenta las condiciones topográficas y climáticas, las barriadas preexistentes y la posibilidad de prolongar algunas vías que arrancaban del interior de la población.

Las zonas previstas se distribuían en un área norte (comprendida por el Hipódromo, la carretera de La Coruña y los límites de la ciudad), sur (circunscrita por Pacífico, las Rondas y el Manzanares), este (entre el Paseo de Ronda y Pacífico), y la oeste (limitada por la carretera de Extremadura, la carretera de Castilla y el Manzanares, entre Puerta de Hierro y el Puente de Segovia). El tratamiento de las cuatro zonas resultaba muy diferente según fueran las condiciones de habitabilidad y la proximidad a los nudos de transporte. Así, la zona norte comprendería una urbanización residencial basada en casas unifamiliares en la Dehesa de la Villa. Toda la zona oeste sería ocupada por la Casa de Campo. Tanto la zona este como la sur parecían ofrecer, en cambio, óptimas posibilidades para el asentamiento de barriadas obreras y de "grandes fábricas y almacenes». El área de Pacífico, las colonias de Fristch y de California, y, en general, los terrenos comprendidos al sur de Delicias, Peñuelas, Gasómetro, Imperial, Marqués de Comillas 
y San Isidro reafirmarían, pues, su naturaleza como primer cinturón industrial de la ciudad.

Se ha señalado la continuidad de criterios expresados en este texto y la idea que guió la materialización del Plan Castro desde mediados del siglo XIX. Recalcando la importancia del tejido viario - ya sea definido por grandes vías, vías radiales y secundarias o por el paseo límite-, se establece como eje del proyecto la necesidad de facilitar los accesos entre el interior y los márgenes del término municipal. El entramado resultante se presenta, así, compacto y ordenado de manera uniforme. En este sentido, y más allá de apreciaciones puntuales, podríamos considerar que plantea un auténtico ensanche desde el cierre del Ensanche ${ }^{34}$. No obstante, hemos de valorar también las innovaciones respecto a lo sugerido cincuenta años antes. No es sólo un mero sistema de alineamiento. En palabras de Ramón López de Lucio se trata del primer plan madrileño de estructura y gestión, donde se desechan además las ortodoxias zonificadoras. Núñez Granés otorga asimismo nuevos contenidos a la propiedad y al Municipio. Aquella debe comprometerse con las urbanizaciones de las vías secundarias. Éste debe promover una expropiación general y urgente. De este modo, el proyecto ha de enmarcarse en una nueva idea de la ciudad, capaz de plantear -a pesar de sus limitaciones- una opción alternativa de gestión frente a los conflictos generados por una suburbanización no regulada ${ }^{35}$.

Por otro lado, el Proyecto también tenía evidentes fines higienistas. En el prólogo firmado por el General José Marvá, maestro de Nuñez Granés, se explicitaba la relación entablada entre este principio y la modernización de la capital, en cuanto fuera capaz de trasladar las «calidades y apariencias de ciudad europea», obteniendo con ello «la cohesión armónica de los diferentes elementos sociales» ${ }^{36}$. En este sentido deben entenderse también los comentarios de prensa publicados en estos meses. Según el cronista José de la Corte, el proyecto de Nuñez Granés favorecería, no tan sólo la edificación de «multitud de barriadas estéticas, cómodas y baratas», sino también el perfil de un futuro "refugio (para) la castigada clase media madrileña, verdadera y única víctima de nuestras luchas sociales» ${ }^{37}$. La urbanización del Extrarradio permitiría, asimismo, rebajar las tensiones generadas por la crisis de la construcción. El Heraldo de Madrid, en julio de 1910, no dudó en relacionar el proyecto con la idea de un «futuro Madrid» donde se resolvieran de manera automática «la higienización (...) de nuestra Metrópoli», «las llamadas crisis obreras» y «la cuestión de las viviendas baratas». Dos años 


\section{La imagen de la ciudad y el paisaje urbano}

más tarde se seguía resaltando la beneficiosa incidencia que el trabajo de Nuñez Granés -entonces ya en vías de tramitación gubernativahabría de tener ante «las míseras condiciones de la vida de Madrid», «la falta de trabajo» y el enquistado conjunto de proyectos parciales planteados desde inicios de siglo ${ }^{38}$.

\section{Notas}

1 Por motivos de espacio hemos optado por reducir al máximo las referencias bibliográficas. En cualquier caso, a partir de la consulta de las obras citadas el lector podrá ampliar los títulos dedicados a este capítulo de la historia urbana madrileña.

2 Cfr. J. C. Rueda Laffond, "Historia social, historia urbana. Aproximación a un modelo de trabajo: la modernización de Madrid en el contexto finisecular, 1890-1914", en Cuestiones de metodología, Bulletin d'Histoire Contemporaine de l'Espagne, 21, VI1995, pp. 95-113.

3 La referencia al paso simbólico de Madrid de Corte a Metrópoli, en J. R. Alonso Pereira, Madrid, 1898-1930. De Corte a Metrópoli, Madrid, CAM, 1985. Como obras generales donde establecer los ritmos históricos del Madrid contemporáneo, A. Bahamonde y L.E. Otero (coords.) La sociedad madrileña en la Restauración, 1876-1931, Madrid, CAM, 1989; A. Fernández (dir.) Historia de Madrid, Madrid, UCM, 1993, pp. 401 y ss.; y S. Juliá (dir.), D. Ringrose y C. Segura, Madrid. Historia de una capital, Madrid, Alianza, 1994. Por su parte, los aspectos abordados en estas páginas han sido analizados con mucho más detalle en nuestra Tesis Doctoral Madrid, 1900. Proyectos de reforma y debate sobre la ciudad, Madrid, UCM, Servicio de Publicaciones, (en prensa).

4 Ayuntamiento de Madrid, Concurso de anteproyectos para la urbanización del Extrarradio y estudio de la reforma interior y extensión de la ciudad. Bases, Madrid, 1929. Sobre el Concurso pueden consultarse también "Programa mínimo a recomendar a los concursantes», La Construcción Moderna, 15-IV-1929, o "Informe del Sr. P. Bonatz, miembro del Jurado en representación de los concursantes extranjeros", Arquitectura, XII-1930.

5 S. Zuazo y H. Jansen, Memoria. Anteproyecto del trazado viario y urbanización de Madrid, s.l., s.f. (pero Madrid, 1930). El entrecomillado pertenece al extracto de una conferencia pronunciada por Zuazo en la Casa del Pueblo de Madrid en el verano de 1931; Arquitectura, IX-1931.

6 Ayuntamiento de Madrid-Oficina de Información, Información sobre la ciudad, Madrid, 1929.

7 Respecto al comportamiento demográfico, A. Fernández, «La población madrileña entre 1876 y 1931. El cambio de modelo demográfico", en La sociedad madrileña..., I, pp. 29-75. El marco secular de la emigración a Madrid, puesto de manifiesto en D.R. Ringrose, Madrid y la economía española, 1560-1850. Ciudad, Corte y país en el Antiguo Régimen, Madrid, Alianza, 1985.

8 A. Gómez Mendoza "La industria de la construcción residencial en Madrid, 1820-1935", en Moneda y Crédito, Madrid, num. 117, pp. 70-75. 
9 Ayuntamiento de Madrid, Población según el Empadronamiento General de Habitantes de 1905, Madrid, 1909; Edificios y habitaciones existentes en la capital según la Estadistica de viviendas formalizada en octubre de 1905, Madrid, 1905; y J. Bravo Ramírez y A. León Peralta Escasez, carestía e higiene de la vivienda en Madrid, Madrid, 1927.

10 Cfr. R. Mas, El barrio de Salamanca. Planteamiento y propiedad inmobiliaria en el Ensanche de Madrid, Madrid, IEAL, 1982.

11 La Ciudad Lineal, la Compañía Madrileña de Urbanización y la figura de Arturo Soria cuentan con abundante bibliografía. Entre los títulos más significativos deben recordarse las aportaciones de R. Collins, C. Flores y A. Soria, Arturo Soria y la Ciudad Lineal, Madrid, Revista de Occidente, 1968; F. de Terán, Soria y la Ciudad Lineal. Antecedente de un urbanismo actual, Madrid, Ciencia Nueva, 1968; D. Brandis y R. Mas «La Ciudad Lineal y la práctica inmobiliaria de la Compañía Madrileña de Urbanización, 1894-1931", en Ciudad y Territorio, 1981, 3; y, muy especialmente, M.A. Maure La Ciudad Lineal de Arturo Soria, Madrid, COAM, 1991.

12 A. Soria, Conferencia dada en el Ateneo Científico y Literario de Madrid el día 14 de mayo de 1894 acerca de la nueva arquitectura de las ciudades, Madrid, 1894, p. 7 .

13 F. Celada y J. Ríos, "Localización espacial de la industria madrileña en 1900", en $L a$ sociedad madrileña..., I, pp. 200-214.

14 A. Gómez Mendoza aborda, en esta misma obra, el papel de Madrid en la red de comunicaciones. Sobre esta cuestión puede consultarse también, del mismo autor, Ferrocarril, industria y mercado en la modernización de España, Madrid, Espasa Calpe, 1989, espec. pp. 133-181; y “Ferrocarril, abastecimientos y mercado nacional: Madrid, 1875-1931", en La sociedad madrileña..., I, pp. 351-375. Acerca de la posición nuclear de Madrid en la red de comunicaciones, A. Bahamonde, L.E. Otero y G. Martínez Lorente, Las comunicaciones en la construcción del estado contemporáneo en España, 1700-1936, Madrid, MOPTU, 1993.

15 Una recapitulación temática y bibliográfica sobre todos estos aspectos, en J. F. Fuentes, "Madrid, ¿paradigma de una historia sociocultural?», en Cercles d'Història Cultural, 1, pp. 12-30.

16 M. Mathet y Coloma, Urbanización de Madrid. Mejoras en el Interior, Madrid, 1903.

17 Para la relación entre reformismo urbano y regeneracionismos, J. C. Rueda Laffond, "Los objetivos políticos regeneracionistas como salida a la crisis del 98", en J. G. Cayuela (ed.) Un siglo de España, 1898-1998. Actas, Cuenca, Universidad de Castilla-La Mancha, 1998.

18 Cfr. C. de Castro, La revolución liberal y los municipios españoles, (1812-1868), Madrid, Alianza, 1979.

19 Sobre esta última cuestión, J. C. Rueda Laffond, «De servicios colectivos a servicios públicos. Propuestas y perspectivas acerca de la municipalización de los servicios urbanos en Madrid, 1890-1914", en Anales del Instituto de Estudios Madrileños, 1996, XXXVI, pp. 533-547.

20 Respecto al paso de Romanones por el Ayuntamiento, véase J. Moreno Luzón, "La corrupción en Madrid: crisis política y regeneracionismo antes del Desastre, (18881898)", en Antes del desastre: orígenes y antecedentes de la crisis del 98, Madrid, UCM,1996, pp. 99-110; y Romanones. Caciquismo y política liberal, Madrid, Alianza, 1998, pp. 67-125. 


\section{La imagen de la ciudad y el paisaje urbano}

21 "Informe del Conde de Romanones", en Ayuntamiento de Madrid, Proyecto de la apertura de una Gran Vía que partiendo de la calle de Alcalá termine en la plaza de San Marcial, Madrid, 1898, pp. 3-6.

22 A. Aguilera, Moción presentada al Excelentísimo Ayuntamiento de Madrid, Madrid, 1902; y Reformas de Madrid. Conferencia dada en el Centro Instructivo del Obrero, Madrid, 1903.

$23 \mathrm{La}$ denuncia de todos estos extremos puede verse, por ejemplo, en S. Moret, «El problema municipal», La España Moderna, T. LXXXIV, 1895, y C. de las Almenas, La municipalidad de Madrid, Madrid, 1896.

24 "Documentos relativos a la Gran Vía», Archivo de Villa, Secretaría, 25-419-2.

25 La Gaceta del Constructor, 14-III-1886 y 13-V-1888; La Ilustración Española y Americana, 8-IX-1901.

26 La idea del ensanche viario, en E. Repullés y Vargas, "Actualidades», Arquitectura y Construcción, IX-1905, pp. 258-260. La expansión del eje Delicias-Prado-Recoletos-Castellana, en J. Grases Riera, Gran Vía Central de Norte a Sur. La mejor calle de Europa en Madrid. Ensanche, higiene, comodidad y belleza, Madrid, 1901.

27 El proyecto inicial en Ayuntamiento de Madrid, Proyecto de la apertura de una Gran Vía que partiendo de la calle de Alcalá termine en la plaza de San Marcial, Madrid, 1898, pp. 3-6. Su posterior concreción, en J. López Sallaberry y F. Andrés Octavio, Mejoras en el interior de Madrid. Memoria del proyecto de saneamiento parcial denominado reforma de la prolongación de la calle de Preciados y enlace de la plaza del Callao con la calle de Alcalá, Madrid, 1904.

28 Ayuntamiento de Madrid, Proyecto de unificación de deudas y obras de saneamiento y mejora de Madrid formulado por el Excelentísimo señor D. Alberto Aguilera y Velasco, Alcalde-Presidente, Madrid, 1906.

29 Ayuntamiento de Madrid, Antecedentes y documentos que comprende el empréstito de liquidación de deudas y obras públicas de treinta y siete millones de pesetas, Madrid, 1908.

$30 \mathrm{El}$ proyecto definitivo puede consultarse en Ayuntamiento de Madrid, Escritura y adjudicación de las obras de reforma y prolongación de la calle de Preciados y enlace de la plaza del Callao con la calle de Alcalá, Madrid, 1910.

31 La Gran Vía, s.l., s.f. (pero París, i192..?).

32 Este texto legal, promulgado el 17 de septiembre de 1896, se encuentra recogido en Ayuntamiento de Madrid-P. Nuñez Granés, El problema de la urbanización del Extrarradio, Madrid, 1919, pp. 417-418. En este volumen se inserta toda la legislación referida a la urbanización del Extrarradio, los proyectos de Ley de 1910, 1914, 1916 y 1918, el proyecto de Nuñez Granés de 1909 y la tramitación del mismo hasta el Real Decreto de 15 de agosto de 1916.

33 La primera edición del trabajo fue publicada bajo el título de Proyecto para la urbanización del Extrarradio de dicha Villa, Madrid, 1910.

34 El propio Núñez Granés insistió en el carácter de Ensanche de su proyecto, si bien en forma de "urbanización periférica». P. Nuñez Granés, "Urbanización del Extrarradio de Madrid», Revista de Municipios, 15-VIII-1910, y "Principios que pueden adoptarse para realizar ensanches urbanos en buenas condiciones económicas», en La Construcción Moderna, 30-VII-1912, pp. 212-217.

35 R. López de Lucio, "Nuñez Granés y la urbanización del extrarradio en el primer tercio del siglo XX», en Ayuntamiento de Madrid, Gestión urbanística europea, 1920-1990, Madrid, Ayuntamiento, 1989. 


\section{José Carlos Rueda Laffond}

322

36 Proyecto para la urbanización..., pp. VII-VIII.

37 J. de la Corte, "Mejoras de Madrid. El plano del Extrarradio", en La Correspondencia de España, 25-V-1910.

38 «La vida en Madrid. Un esfuerzo necesario», en El Imparcial, 11-IX-1912. 